\title{
La mujer en la empresa familiar española desde la perspectiva del familiness ${ }^{1}$
}

\author{
Pilar ORTIZ GARCÍA \\ Universidad de Murcia \\ portizg@um.es \\ Ángel José OLAZ CAPITÁN \\ Universidad de Murcia \\ olazcapi@um.es
}

Recibido: 06-12-2013

Aceptado: 04-06-2014

\section{Resumen:}

La mujer es una de las piezas clave en la transmisión de valores a la familia y a la empresa familiar. Este trabajo analiza la contribución de la mujer a la formación de valores en la empresa familiar. La perspectiva de análisis a partir del concepto de familiness, permite identificar el papel de la mujer en las dimensiones de poder, experiencia y cultura que componen el "capital familiar" de la empresa. La metodología de análisis se ha basado en la explotación de una encuesta a 282 empresas familiares en toda España. Los resultados ponen de manifiesto la participación de las mujeres en la formación de familiness en sintonía con el papel atribuido a la mujer en la familia y en la empresa.

Palabras clave: roles de género, empresa familiar, capital social, familiness, valores, socialización

${ }^{1}$ Este estudio forma parte de la participación de los autores en el proyecto: “El éxito de la empresa familiar: la relación entre el negocio y la familia desde la perspectiva del familiness” financiado por el Ministerio de Ciencia e Innovación con referencia CSO2010 - 17761 


\title{
Women in the spanish family business from the perspective of familiness
}

\begin{abstract}
The woman is one of the key pieces in the transmission of values to the family and the family business. This paper analyzes the contribution of women to the formation of values in the family business. The analytical perspective from familiness concept, to identify the role of women in the dimensions of power, experience and culture that make up "family capital" of the company. The analysis methodology has been operating a family business survey in Spain. The results demonstrate the participation of women in training familiness in tune with the role assigned to women in the family and in the company
\end{abstract}

Keywords: gender roles, family business, social capital, familiness, values, socialization

\section{Referencia normalizada}

Ortiz García, P., Olaz Capitán, A.J. (2014). "La mujer en la empresa familiar española desde la perspectiva del familiness”. Política y Sociedad, Vol 51, Núm. 2: 481-506

Sumario: Introducción. 1.Roles de la mujer en la empresa familiar. 2.Mujer y familiness. 3. Metodología. 4.Resultados. 5. Conclusiones 


\section{Introducción}

El papel de la mujer en la empresa discurre en paralelo con su presencia en el ámbito público. En el caso de España, la participación de las mujeres en ámbitos de poder y toma de decisiones en la política (órganos de dirección de partidos, Congreso, Senado) aunque ha evolucionado, en la actualidad se sitúa en torno al treinta por ciento $^{2}$. En la empresa privada la participación es todavía más discreta. Los últimos datos del Instituto de la Mujer señalan que en 2009 un 10,3\% de mujeres ocupaba algún cargo en el consejo de administración de las empresas del IBEX 35; tratándose, por consiguiente, de un porcentaje muy bajo, aunque alentador si se considera que cuatro años antes eran tan sólo de un 2,1\%.

La mujer sigue siendo especialmente visible en el ámbito privado, aunque no renuncia a su participación en el público. Los roles tradicionalmente asignados a la mujer, siguiendo un esquema de división sexual del trabajo o especialización funcional, legitiman su presencia en el ámbito privado. Sin embargo, la presencia de la mujer en el ámbito público, al que pertenece el trabajo, adquiere con frecuencia la consideración de actividad complementaria, lo que explica una mayor irregularidad en las trayectorias laborales de las mujeres (Carrasquer, 2009). La teoría de género ha puesto de manifiesto que la articulación entre el trabajo productivo y el reproductivo lleva a las mujeres a una suerte de "doble presencia" (Balbo, 1978), o doble jornada (Tobío, 2002; 2005; Papí y Frau, 2005; Tobío et al., 2010, Carrasquer et al., 1998; Torns, 2005), que se traduce, más que en una conciliación, en la "acumulación” de ambos ámbitos (Maruani, 2002).

Se trata, por tanto, de un problema estructural en el mercado laboral alimentado por un aspecto cultural que apunta a las creencias, los prejuicios y algunos valores también presentes en la empresa y en la familia y, cómo no, en esta estructura simbiótica de ambos elementos que es la empresa familiar.

En este sentido, la literatura en torno al papel de la mujer en la empresa y en concreto en la empresa familiar, no abunda, como tampoco los estudios empíricos sobre el tema. Pese a ello, desde finales de los años ochenta se han publicado una serie de investigaciones que abordan la cuestión. Entre ellas, cabe establecer dos grandes grupos. Un primer grupo de estudios ha estado centrado en explicitar las dificultades de la mujer para acceder a un ámbito hasta ahora bastante mediatizado por el poder masculino y en el que resulta difícil el reconocimiento a su contribución (Dumas, 1989; Lyman et al., 1985; Salganicoff, 1990; Fitzgerald y Muske,

${ }^{2}$ Según los datos del Instituto de la Mujer, en la legislatura 2011-2015 hay un 36\% de mujeres diputadas. En los parlamentos autonómicos ese porcentaje asciende al $43,22 \%$ en 2012. En la misma legislatura, en el Senado hay un 33,3\% de mujeres. En 2011 el porcentaje de mujeres en el Gobierno es de 30,7\%. También hay un 31,5\% de mujeres Delegadas de Gobierno en provincias y Comunidades Autónomas. 
2002). En la misma dirección, algunos estudios adoptan un carácter reivindicativo poniendo el acento en la brecha existente entre la participación de la mujer en aspectos como la sucesión ligada al desarrollo de la carrera profesional (Martínez et al., 2007) y las cotas de poder o de propiedad en la empresa familiar (Dumas, 1998).

Un segundo grupo de estudios pone de manifiesto las singularidades de la mujer en la empresa familiar y su aportación como un recurso competitivo en las empresas de este carácter. En este último sentido, el estudio de la visibilidad de la mujer y su papel en procesos clave de la empresa familiar, como son la propiedad, la gestión y la transición/sucesión, así como la peculiaridad de su actuación en función del doble rol que desempeña en la familia y en la empresa, han sido los temas tratados (Cole, 1997; Rowe y Hong, 2000; Sharma, 2004; Vera y Dean, 2005; Vadnjal y Zupan, 2009).

Esta investigación abunda en el segundo grupo de análisis al abordar la variable género en la empresa familiar desde la perspectiva del familiness. Esta perspectiva, si bien ha tenido un desarrollo aceptable en el ámbito científico internacional (Habbershon et al., 2003; Chrisman et al., 2005; 2008; Habbershon, 2006; Danes et al., 2007), no ha tenido un progreso equivalente en los estudios sobre empresa familiar en España.

Con el objeto de clarificar este enfoque, así como el objeto de estudio, resulta necesario definir los conceptos de familiness, capital familiar y valores familiares.

El origen del término familiness está en la teoría de recursos y capacidades. Dicha teoría indica que la combinación de recursos y capacidades de una empresa es su principal ventaja competitiva (Haberson y Williams, 1999). En este contexto, Olson et al., (2003: 452) y Chrisman et al., (2005) se refieren al familiness como los recursos y capacidades resultantes de la implicación de la familia en la empresa y las interacciones a que dicha implicación da lugar.

El familiness alude a la idiosincrasia de la empresa resultante de la combinación entre el sistema familia y el sistema empresa (Habbershon et al., 2003). Los estudios sobre el tema inciden en cómo la influencia familiar es susceptible de crear valor y ser una ventaja competitiva - como un capital más - en la empresa familiar (Pearson et al., 2008; Olson et al., 2003; Cibrián, 2010).

Esta influencia se concreta en tres dimensiones en el modelo de Astrachan et al. (2002): el poder de la familia en la empresa (accionariado y participación en órganos directivos); la experiencia que la familia aporta al negocio transmitida de generación en generación y la cultura, esto es, los valores, actitudes y motivaciones que imperan en la empresa. Se trata de las dimensiones de la escala F-PEC (power, experience y culture) utilizada como modelo metodológico en este estudio.

La dimensión cultural del familiness se identifica con el "capital familiar" o capital social familiar; un capital que se define, siguiendo a Hoffman, et al. (2006), como la "infraestructura moral" (entendiendo por tal los valores, normas y creencias moralmente aceptadas) en la que se socializan los miembros de la unidad familiar, que dirige las relaciones entre los miembros de la familia y a través del cual la familia empresaria aprende a trazar el tipo de empresa que quiere ser. Los valores familiares enraizados en la organización son todo aquello que la organiza- 
ción considera importante y el principal componente de la variable cultura en el modelo de análisis de la empresa familiar desde el familiness.

Esta orientación abre una interesante perspectiva sobre el papel de la mujer en la empresa familiar, dada su posición como una de las piezas clave en la transmisión de valores en la familia. En este sentido, el objetivo del artículo es realizar una aproximación a la empresa familiar desde la perspectiva familiness, enfatizando en la posición de las mujeres y su percepción sobre la influencia de la familia en la empresa. En segundo lugar, se trata de determinar en qué medida dicha percepción se ve condicionada por los roles de género en la familia y en la empresa.

Desde el comienzo de la crisis, la subida de la tasa de paro en España ha sido muy intensa. Sin embargo, el comportamiento de esta variable ha sido distinto entre hombres y mujeres. A partir de la Encuesta de Población Activa (EPA) se puede constatar que en el período comprendido entre 2006 a 2010, la tasa de empleo de los varones de 16 y más años disminuyó 10,1 puntos y la de las mujeres también lo hizo, aunque en solo 0,8 puntos. Este descenso es especialmente acusado entre la población más joven (de 16 a 24 años) no obstante, es significativamente mayor en el caso de los varones (20,3 puntos porcentuales en el caso de los hombres y 11,2 puntos en el caso de las mujeres) para este mismo período. Esto ha provocado una paulatina disminución en la brecha de género, aproximándose al valor que representa dicho indicador en la UE-27 que en 2010 ascendía a 11,9 puntos. Igualmente, la brecha de género en la tasa de paro se ha reducido como consecuencia del fuerte incremento de esta tasa entre la población masculina y el mejor comportamiento de la tasa de paro femenina en este momento de la crisis.

Estos datos contextualizan la participación de la mujer en el mercado de trabajo y su aproximación a la del hombre. Sin embargo ¿dónde se empiezan a establecer las diferencias? En 2010 el 77,7\% de las mujeres ocupadas eran empleadas (con jefes y sin subordinados) y el 8,2\% eran ocupadas independientes (sin jefes ni subordinados). Sólo el 0,3\% eran directoras de empresa grande o media. En resumen, a medida que se asciende en el grado de independencia y responsabilidad en el puesto, decrece la participación femenina en dichas ocupaciones. La literatura reciente sobre el tema en el ámbito nacional (Gisbert et al., 2009; Mateos et al., 2009; Peris - Ortiz et al., 2010), como internacional (Hwelett, 2002; Burke, 2007) pone de manifiesto las menores oportunidades de las mujeres hacia puestos que impliquen responsabilidad y toma de decisiones.

Estos datos dimensionan la actividad empresarial de la mujer y sirven de marco explicativo sobre el rol desempeñado en la empresa familiar. Un papel en el que se reproducen las diferencias de género construidas en el ámbito laboral y familiar.

\section{Roles de la mujer en la empresa familiar.}

La integración de la perspectiva de género en el análisis del empleo es importante para entender cómo éste no se distribuye de forma "neutral". En este contexto, se alude al género como una construcción sociocultural que 
atribuye cualidades, funciones y espacios -a la mujer- que determinan su posición en el mercado de trabajo. Se trata de un hecho que tiene su origen en el ámbito familiar.

Cuando se indaga en la literatura sobre el papel de la mujer en la empresa familiar se constata una escasez de estudios empíricos en la que existen pocas excepciones (Rowe y Hong, 2000; Dumas, 1998; Bowman - Upton y Hechk, 1996). Básicamente se abren dos vías en las que la literatura aborda el tema (Martínez et al., 2007). Un primer grupo de trabajos pone de manifiesto las dificultades de la mujer para incorporarse a la empresa y el desarrollo de una carrera profesional una vez lo han conseguido, o los problemas de reconocimiento del trabajo (Lyman et al., 1985; Dumas, 1989; Salganicoff, 1990). En segundo lugar -y desde una perspectiva posibilista-, otros estudios analizan el desempeño de la mujer en la empresa familiar y su competencia para ostentar cargos de responsabilidad en la gestión de ésta (Cole, 1997; Rowe y Hong, 2000; Vera y Dean, 2005). Estos estudios enfatizan en aspectos asociados a la sociabilidad, la observación, el capital emocional (Sharma, 2004) y, en general, el despliegue de habilidades sociales que facilitan el desempeño, aunque no están relacionadas directamente con la consecución de objetivos (un rol asociado al varón).

Con respecto a la primera vía de análisis los estudios que identifican las dificultades en el reconocimiento del papel de la mujer en la empresa familiar, ponen el acento en aspectos relacionados con los estereotipos asociados al género. En este sentido, el papel de la mujer está ligado, no tanto a la valoración de su labor, como a la recompensa psicológica que ésta recibe por la ayuda a una "empresa" común. Aspectos tales como la cercanía al negocio están por encima de la propiedad formal que, a veces, pasa a un segundo plano frente a la "propiedad psicológica" y el compromiso que las hace concebir la empresa como parte de su familia (Heinonen y Stenholm, 2011).

En el mismo sentido, aunque teniendo en cuenta en este caso la posición de la mujer en la estructura familiar, el papel de la mujer-esposa del fundador de la empresa, hija, hermana o nuera de éste, puede ser muy distinto. La literatura se centra especialmente en el rol desempeñado por las hijas, ligado a la cuestión de la sucesión. Las dudas sobre su capacidad de compromiso a largo plazo las relegan a un papel secundario, optando a la sucesión en el liderazgo cuando no existe un descendiente varón (Gallo, 1995). La investigación sobre el tema pone también de manifiesto que la socialización de género opera fuertemente en la asunción de liderazgo en la empresa familiar (Iannarelli, 1992).

No obstante todo ello, abundando en la vía que identifica los activos de la mujer en la empresa familiar, investigaciones recientes evidencian que la presencia de la mujer en los puestos directivos de las empresas familiares es más frecuente que en las empresas que no tienen dicho carácter (Mateos et al., 2006; Rodríguez y Rodríguez, 2011). Ello es debido a que la pertenencia a la familia, elimina gran parte de las barreras de acceso a este tipo de puestos. El reclutamiento interno forma parte del proceso de aprovisionamiento de puestos en las empresas familiares, lo que facilita el camino de la mujer. Por otra parte, recientes investigaciones ponen de 
manifiesto que la mujer se mueve por el compromiso, la lealtad y los valores de la familia, lo que las convierte en candidatas especialmente idóneas para ocupar puestos de responsabilidad en la empresa (Rodríguez y Rodríguez, 2011).

En el caso que nos ocupa se analiza la posición de la mujer con responsabilidades directivas en la empresa familiar, ya que el análisis parte de su percepción sobre la influencia de la familia en la empresa.

\section{Mujer y familiness.}

Los trabajos empíricos realizados sobre la influencia de la familia en la empresa familiar han permitido desarrollar un concepto interesante: el de capital social. Este concepto surge de la sociología (Coleman, 1988; Putnam, 1995), aunque será considerado como variable de análisis en el ámbito económico, tanto en los análisis de empresa, como en todos aquellos relacionados con desarrollo económico. Se puede definir capital social como la creación de valor (capital) derivado de la relación entre los individuos (social). De la misma forma que el trabajo productivo se traduce en la generación de bienes, o el capital humano se traduce en conocimiento, las relaciones sociales pueden tener un efecto en términos de beneficio, aunque tampoco puede obviarse las dificultades propias de su medición.

Coleman (1988) identifica algunos elementos clave de dicho capital. En primer lugar, se refiere a las redes sociales, como son los lazos de parentesco, las redes comunitarias informales y las organizaciones sociales.

La empresa familiar es un tipo de organización en el que los principios enunciados por Coleman encuentran un soporte idóneo para su desarrollo. Los vínculos sociales están garantizados a través de la relación de parentesco; las normas u obligaciones vienen establecidas en una doble clave: la económica (trabajo y aportación económica) y la familiar (dedicación de la familia al desarrollo de la empresa, el negocio como proyecto familiar...). Por último, la confianza, forma parte de la propia naturaleza de la familia y, por extensión, de las relaciones económicas establecidas en este tipo de empresa. Por tanto, como presupuesto inicial, la empresa familiar es uno de los tipos de organización en la que las relaciones sociales son especialmente susceptibles de generar valor.

El "familismo" resulta de la potencialidad del capital social familiar para generar beneficios empresariales y no solamente económicos. Uno de los elementos distintivos de las empresas familiares consiste en que sus objetivos trascienden los aspectos puramente económicos (Olson et al. 2003; Chrisman et al. 2008; Miller et al. 2008; Sharma, 2004; Hienerth y Kessler, 2006; Kellermanns et al., 2008 y Cibrián, 2010). La preocupación por las generaciones futuras y el mantenimiento del equilibrio emocional de la familia, a partir de una buena gestión de los recursos en la empresa, son factores importantes en este tipo de empresas. Son varios los marcos teóricos desde los que se aborda esta relación: la Teoría de Sistemas (Lansberg, 
1983), la Teoría de Recursos y Capacidades (Habbershon et al., 2003), o la del Capital Social (Coleman, 1988).

Como se definió con anterioridad, el capital familiar es una especial forma de capital social; se trata de la infraestructura moral que dirige las relaciones entre los miembros de la familia. Del desarrollo de dicho capital puede depender, en gran medida, el que la influencia de la familia sea positiva o negativa en la empresa. Un capital familiar funcional -entendiendo por éste el que impulsa aspectos relacionados con el compromiso, la comunicación, el trabajo en equipo-, será adecuado para el desarrollo de la empresa. Por el contrario, un capital familiar disfuncional tendrá capacidad para "contaminar” su desarrollo (Le Breton-Miller y Miller, 2009; Sorenson, R. y Bierman, L., 2009; Cibrián, 2010).

Teniendo en cuenta estos conceptos ¿cuál es el papel de la mujer en la generación de este tipo de capital relacional que posibilita ventajas competitivas a través del valor de las relaciones sociales? La respuesta debe hallarse en los roles desempeñados por ésta en la familia como generadora de vínculos emocionales y cultura, así como en la ascendencia de dichos vínculos y valores sobre la empresa, dado que, a menudo, los roles de género que imperan en la familia empresaria se trasladan a la empresa familiar (Ceja, 2008). Los estudios enfatizan en el incuestionable desempeño profesional de la mujer cuando adquiere posiciones de poder; pero, al mismo tiempo, cumple por la educación generalmente recibida-, un importante papel en el mantenimiento del equilibrio y la armonía emocional de la familia.

Las investigaciones sobre el tema (Lansberg, 1995; Rowe y Hong, 2000; Ceja, 2008; Sharma y Nordqvist, 2008) atribuyen a la mujer una serie de funciones en la empresa familiar. En primer lugar, la transmisión de valores. Por su rol tradicional en la familia, la mujer tiene la oportunidad de generar, transmitir y reforzar los valores que conforman el capital cultural de ésta. En segundo lugar, la educación generacional en estos valores que conforman la personalidad de los sucesores en la empresa. Por último, el liderazgo emocional. En este sentido, algunos estudios muestran que las tareas relacionadas con la promoción del compromiso de la familia hacia la empresa, suele ser desarrollado por las mujeres, así como la promoción de la unión, la armonía y el equilibrio emocional de la familia ${ }^{3}$. El conocimiento acumulado por la

\footnotetext{
${ }^{3}$ El estudio referido por Ceja (2008) realizado por la Cátedra de Empresa familiar IESE en 2006 se basó en 200 entrevistas a miembros no activos de 98 empresas españolas y en él se trataba de estudiar la existencia de una posible división de roles por género en la empresa familiar.
} 
mujer en esta materia la "dota” de una especial inteligencia emocional, que sería útil en la elaboración de aquellos órganos de regulación de las relaciones familiares en la empresa, como es el Consejo de Familia o la promoción de reuniones intergeneracionales, necesarias para conservar el equilibrio entre la esfera familiar y la empresarial a partir del fomento de la comunicación.

Por otra parte, existe una convicción acerca del desarrollo de determinadas cualificaciones tácitas en la mujer que se han instalado en la conciencia colectiva y han sido interiorizadas por ésta. Atributos que van desde los aspectos físicos tales como "meticulosidad", hasta los inmateriales y subjetivos como la "lealtad”, la “flexibilidad”, la "paciencia” o la "atención”. Estas cualificaciones -construidas socialmente-, son generalmente infravaloradas, ya que a diferencia de las cualificaciones tradicionalmente masculinas (conocimiento, experiencia) adquiridas en el sistema educativo o productivo, las de las mujeres se adquieren en el sistema informal que es la familia, a través de la socialización y son menos susceptibles de medición, razones por las cuales se consideran al margen del ámbito de generación de valor (Rigby y Sanchís, 2006). No obstante en este caso, dichas cualificaciones podrían constituir un importante activo en la gestión de la empresa familiar.

\section{Metodología.}

Este estudio se realizó en un total de 500 empresas pequeñas y medianas de toda España de las cuales 282 tienen un carácter familiar. Se define empresa familiar como aquella en la que la mayoría de los votos son propiedad de la persona o personas de la familia que fundó la compañía (participación mayoritaria en la propiedad) y al menos un representante de la familia o pariente participa en la gestión o gobierno de la compañía (participación en la gestión) ${ }^{4}$. El sistema de recogida de información fue mediante entrevista telefónica grabada a través de sistema CATI a los principales responsables de la empresa (Director General, Gerente, Director de Recursos Humanos y puestos afines). El trabajo de campo se desarrolló entre los días 28 de febrero y el 1 de marzo de 2011.

Para la realización de este trabajo se empleó un marco muestral de la base OSIRIS (del Bureau Van Dyck Electronic Publishing) compuesto por un total de 5.113 empresas de toda España, cuyo número de trabajadores estaba comprendido entre los 25 y los 249 empleados. La muestra se segmentó atendiendo al tamaño de

\footnotetext{
${ }^{4}$ Definición propuesta por el European Group of Owner Managed and Family Enterprises (GEEF) y por la Family Business Network (FBN) y adoptada por el Instituto de Empresa familiar.
} 
la empresa, de manera que se realizaron 200 encuestas en empresas de entre 25 y 49 trabajadores, y 300 encuestas entre empresas de 50 a 249 trabajadores. Finalmente y a efectos concretos de este trabajo-, la muestra se concreta en 282 empresas familiares. El procedimiento de depuración de las empresas familiares de las no familiares consistió en la pregunta planteada a la empresa sobre si ésta es considerada familiar o no atendiendo a los criterios anteriormente establecidos para la definición de una empresa de este carácter (cuestión a la que respondieron afirmativamente un 56,4\%).

El error muestral fue de un $\pm 4,25 \%$, considerando el UOE al número de empresas facilitadas en el marco muestral utilizado (5.113) y asumiendo criterios de muestreo aleatorio simple para el caso de máxima indeterminación [P (probabilidad del fenómeno $)=q($ probabilidad complementaria $)=50 \%]$ y un nivel de confianza del 95,5\% $(\mathrm{k}=2)$. La selección de la unidad muestral se realizó siguiendo un proceso aleatorio sistemático a través de llamadas telefónicas.

El cuestionario estructurado constó de 18 preguntas referidas a la situación de políticas fiscales, recursos humanos e innovación. La depuración de la matriz de datos se realizó a través de los programas BARWIN, CODI, MINITAB, EXCEL y SPSS. En cuanto al modelo utilizado para dimensionar la influencia de la familia en la empresa y su repercusión sobre la formación de capital familiar, se ha utilizado la escala F - PEC ${ }^{5}$ (Power, Experience, Culture) de Astrachan et al. (2002).

De acuerdo con el interés de este análisis, se utiliza la subescala de cultura ${ }^{6}$ del modelo. Esta dimensión considera en qué grado los valores de la familia están conectados con los valores de la empresa y, en segundo lugar, qué grado de compromiso tiene la familia con la empresa.

La distribución muestral en razón de sexo limita la posibilidad de realizar análisis estadísticos de otro carácter que no sea el exploratorio y descriptivo. Asimismo, no se trata tanto de realizar un análisis comparativo entre hombres y mujeres, sino de realizar una aproximación al familiness introduciendo la variable género.

Esta aproximación permite plantear la cuestión de investigación, esto es, tal como pone de manifiesto la teoría sobre el tema, se trata de determinar si la construcción sexuada de roles en la familia y en la empresa influye en alguna medida en el "familismo" (poder de la familia, experiencia de la familia y cultura).

Para ello se observará la participación de la mujer en la empresa familiar, en sus órganos de gobierno y puestos directivos (dimensión poder). Se trata de una posi-

${ }^{5}$ Es introducida por Astrachan , Klien y Smyrnioff para medir la influencia de la familia en la empresa desde tres perspectivas: el poder, la experiencia y la cultura. Cada factor constituye una subescala que agrupa las variables consideradas significativas para determinar la el grado de influencia familiar en el negocio.

${ }^{6}$ Corresponde al apartado 2 del cuestionario, preguntas 9 y 10. 
ción en la que habitualmente la mujer desempeña un rol secundario (hija o esposa), reproduciendo así la tesis que apunta al papel de catalizadora de emociones, propiciadora de consenso y compromiso con el proyecto empresarial y, en general, el rol de colaboración frente al de competencia (Ceja, 2008; Gallo, 1995). La dimensión experiencia de la familia se analiza a partir de la generación que controla la empresa y, por último, la dimensión cultura -objeto fundamental del análisis- a partir de los componentes de esta: conexión de valores y grado de compromiso entre la familia y la empresa.

\section{Resultados.}

A continuación se analizan los resultados de la encuesta tomando como referencia las 282 empresas familiares. En un intento por sintetizar aquellos aspectos que permitirían caracterizar algunas singularidades del estudio, comenzaremos por decir que las personas que han realizado la encuesta tienen responsabilidad en ella, ya sea en tareas de dirección, gerencia o en la propiedad de la empresa. De todos ellas, un $7,1 \%$ son mujeres frente a un 92,9\% de hombres. Esta distribución pone de manifiesto la infrarrepresentación de las mujeres con responsabilidades directivas en el ámbito empresarial familiar, especialmente a partir de empresas de un cierto tamaño, como es el caso, según se ha especificado con anterioridad en el marco muestral.

Dicha representación está en la misma dirección de estudios previos que indican la menor experiencia directiva y empresarial de la mujer, un hecho que se reproduce también en la empresa familiar (Peris-Ortiz et al., 2010; Meroño y López, 2012). Como se extrae de los resultados de la encuesta, el perfil de la mujer en las empresas familiares analizadas es el de personas con una antigüedad media en la dirección o gerencia de algo más de 13 años (sensiblemente inferior a los varones que está por encima de los 33 años). En lo referente a la formación, la de las mujeres es superior a la de los hombres, concretamente un 70\% tienen formación universitaria frente al $63,4 \%$ de hombres.

\section{Dimensión poder}

En cuanto a la dotación de determinados órganos de gobierno, la creciente complejidad de la empresa familiar ha conllevado una paulatina profesionalización en la gestión. El hecho de que la mayoría de las empresas $(66,7 \%)$ estén dotadas de un Consejo de Administración (Gráfico 1) debe entenderse como una práctica de buen gobierno que garantiza la continuidad del negocio familiar en el tiempo, a la vez que facilita el gobierno y organización de la empresa (Carrasco y Sabater, 2009; Mustakallio et al., 2002; Corona, 2005). 
Gráfico 1. Empresas familiares según composición del Consejo de Administración y sexo del gerente (\%)

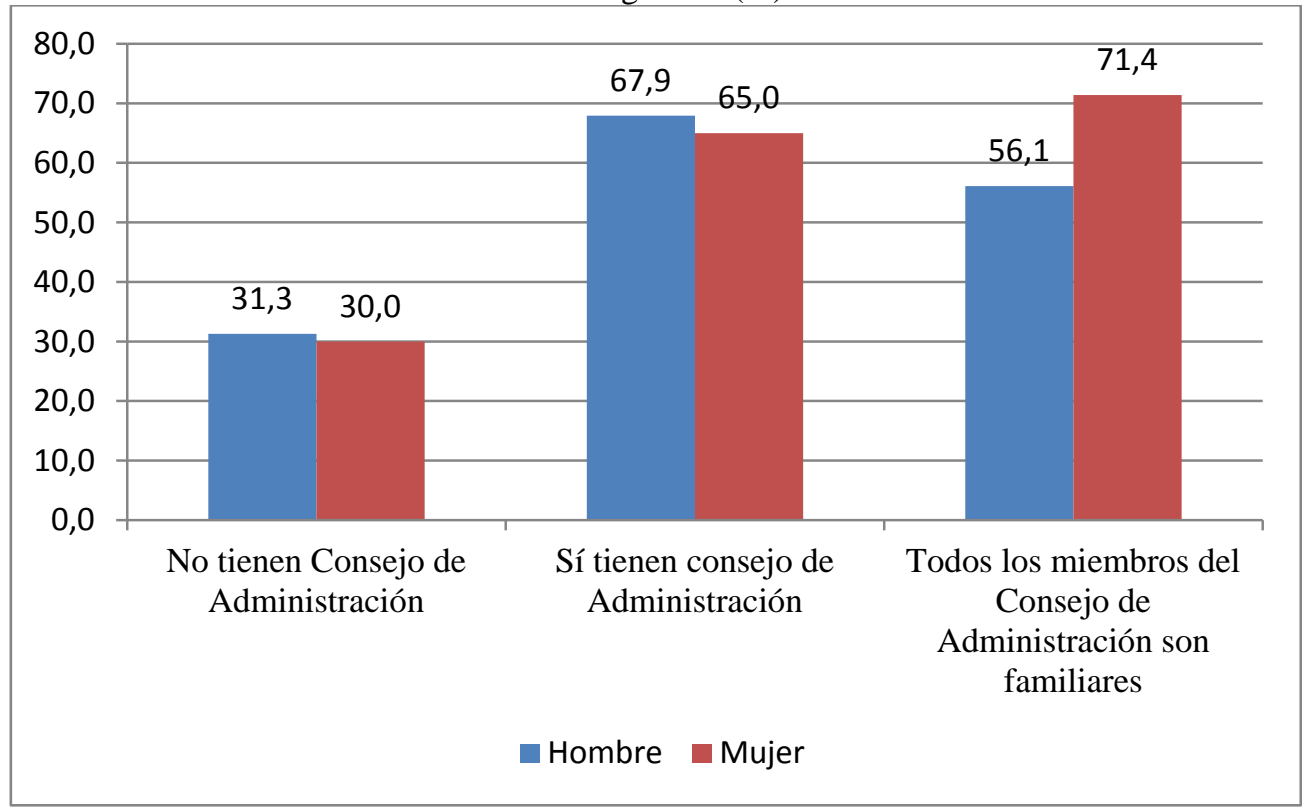

Fuente: Encuesta a empresas familiares, 2011.

La variable género no resulta determinante en la dotación de órganos de gobierno como es el Consejo de Administración, si bien, se observa una ligera diferencia en cuanto la composición de este órgano de gobierno en el caso de las empresas familiares dirigidas por mujeres (65\%), respecto a las empresas dirigidas por hombres (67,9\%). Así, en las lideradas por mujeres (y que disponen de dicho órgano de gobierno), un 71,4\% de los miembros del Consejo de Administración pertenecen a la familia; mientras que en las dirigidas por sus homólogos varones, este porcentaje se reduce al 56,1\%. La participación de la familia en este órgano de dirección de la empresa - uno de los componentes del familiness - cobra una especial importancia en los negocios dirigidos por mujeres.

Abundando en la relevancia que adquiere la familia en la dirección en empresas lideradas por mujeres, el dato mencionado anteriormente cobra mayor sentido si se tiene en cuenta que en éstas, un 55\% de los puestos directivos están ocupados por miembros de la familia, mientras que en las empresas en las que el gerente es un hombre, la familia ocupa sólo el 28,6\% de los puestos de dirección.

\section{Dimensión experiencia}

Esta dimensión se refiere a la experiencia que la familia aporta al negocio a través de las generaciones que participan o han participado en la empresa. Resulta particu- 
larmente interesante desde la perspectiva cultural, dado que la cultura de la empresa familiar aparece ligada a los valores del fundador/a (Ortiz et al., 2009).

En la empresas analizadas, existe una menor proporción de empresas en primera generación dirigidas por mujeres (en concreto, un 40\%), que dirigidas por hombres $(45,4 \%)$. Se trata de un dato coherente con la menor experiencia directiva de las primeras, como se ha constatado anteriormente. No obstante, resulta de interés reparar en algunas diferencias sobre la propiedad y la dirección en función de la generación que controla la empresa. Si se observa los datos de la tabla 1, se aprecia la escasa diferencia respecto a la propiedad en manos de la generación fundadora (en el caso de los hombres está en esta generación en el 46,8\% de los casos y en el caso de la mujer en el 50\%), sin embargo, cuando se habla de dirección, aparece algún desequilibrio dependiendo del sexo, en este caso, la mujer directiva ya no pertenece mayoritariamente a la generación fundadora (60\% de las mujeres directivas encuestadas en "otra" generación de la empresa frente a 54,6\% de hombres), sino a "otra" (segunda o sucesivas). Este dato es coherente con los estudios sobre el tema que evidencian el papel secundario de la mujer en el acceso a la dirección, a la que accede cuando el fundador - generalmente varón-, ha cedido la dirección (Dumas, 1998) y entra en segunda generación o sucesivas.

Tabla 1. Generación que controla la propiedad y la dirección en la empresa familiar, y sexo

\begin{tabular}{|l|c|c|c|c|c|c|c|c|}
\hline \multicolumn{2}{|c|}{} & \multicolumn{4}{c|}{ Propiedad } & \multicolumn{3}{c|}{ Dirección } \\
\hline & \multicolumn{2}{|c|}{ Hombre } & \multicolumn{2}{c|}{ Mujer } & \multicolumn{2}{c|}{ Hombre } & \multicolumn{2}{c|}{ Mujer } \\
\cline { 2 - 10 } & $\mathrm{N}$ & $\%$ & $\mathrm{~N}$ & $\%$ & $\mathrm{~N}$ & $\%$ & $\mathrm{~N}$ & $\%$ \\
\hline Fundadora & 95 & 46,8 & 8 & 50 & 119 & 45,4 & 8 & 40 \\
\hline Otra & 108 & 53,2 & 8 & 50 & 143 & 54,6 & 12 & 60 \\
\hline Total & 203 & 100 & 16 & 100 & 262 & 100 & 20 & 100 \\
\hline Fuente: Encuesta a empresas familiares, 2011. & & & & & \\
\hline
\end{tabular}

Esta dimensión contextualiza el papel de la mujer en otro de sus roles en la empresa familiar, como es el de hija/mujer o, en definitiva, la persona que no ocupa un papel principal como fundadora/directora. La posición de la mujer en estos casos abunda en la función armonizadora y conciliadora, dada una educación en la que, frente al posible hijo varón, su rol no es el de competencia frente al padre/fundador, sino el de colaboración (Gallo, 1995; Leach, 1999; Ceja, 2008).

No son pocos los análisis que enfatizan en las relaciones padre-hija y en cómo esta vinculación se suele caracterizar por una mayor complementariedad en el trabajo que la producida con los hijos varones (Dumas, 1992; Kets de Vries, 1996).

Según esta primera aproximación a las características de las empresas familiares que están lideradas por mujeres, se observa que contienen un mayor nivel de "familismo" en sus órganos de gobierno y puestos directivos que las dirigidas por hombres. Ello es susceptible de potenciar la función de la mujer como transmisora de 
valores y su papel como agente socializador en la empresa familiar. Por otra parte, en cuanto a la experiencia generacional, por regla general accede a la dirección en segunda generación o sucesivas.

\section{Dimensión cultura}

Una vez identificada la posición de la mujer en las dimensiones poder y experiencia del modelo familiness, la dimensión cultura se refiere al conjunto de valores, motivaciones y actitudes que imperan en la empresa, y en qué medida se perciben como el resultado de una conjunción entre el negocio y la familia en términos de compromiso, lo que compone un capital familiar susceptible de constituir una ventaja competitiva para la empresa de este carácter.

En esta apartado la variable de género resulta de interés para realizar una aproximación a la percepción de la mujer sobre esta dimensión del familiness, y observar en qué medida dicha percepción se ve condicionada por los roles de género en la familia y en la empresa.

Tomando el sexo del gerente como variable independiente, y como variables dependientes las que permiten una identificación del familiness en su dimensión cultural según la escala F-PEC de Astrachan et al. (2002), se ha realizado un análisis ANOVA de comparación de medias de respuesta por sexo en la escala establecida (de 1 a 5 en la que 1 es "Total desacuerdo" y 5 es "Total acuerdo") obteniéndose los resultados que se exponen en la tabla 2.

Tabla 2. Percepción sobre la influencia de la familia propietaria en la empresa familiar

\begin{tabular}{|l|c|c|c|c|c|c|}
\hline & \multicolumn{2}{|c|}{ Hombre } & \multicolumn{2}{c|}{ Mujer } & \multicolumn{2}{c|}{ Total } \\
\hline & $(\mathrm{N})$ & Media & $(\mathrm{N})$ & Media & $\mathrm{N}$ & $\begin{array}{c}\text { Me } \\
\text { dia }\end{array}$ \\
\hline Tiene influencia en el negocio & 261 & 4,6 & 20 & 4,6 & 281 & 4,6 \\
\hline $\begin{array}{l}\text { Comparte valores similares en el } \\
\text { negocio }\end{array}$ & 251 & 4,5 & 19 & 4,6 & 270 & 4,5 \\
\hline Se siente orgullosa del negocio & 258 & 4,8 & 20 & 5,0 & 278 & 4,8 \\
\hline Se preocupa por el destino del negocio & 259 & 4,8 & 20 & 5,0 & 279 & 4,8 \\
\hline $\begin{array}{l}\text { Entiende y defiende las decisiones que } \\
\text { afectan al futuro de la empresa }\end{array}$ & 259 & 4,7 & 19 & 4,8 & 278 & 4,7 \\
\hline $\begin{array}{l}\text { Sabe diferenciar los aspectos empresa- } \\
\text { riales de los familiares }\end{array}$ & 253 & 4,1 & 20 & 4,6 & 273 & 4,2 \\
\hline $\begin{array}{l}\text { Se ve afectada por los conflictos que se } \\
\text { producen en la empresa }\end{array}$ & 251 & 3,6 & 20 & 3,8 & 271 & 3,6 \\
\hline $\begin{array}{l}\text { Con sus valores familiares contribuye a } \\
\text { generar un buen clima en la empresa }\end{array}$ & 257 & 4,1 & 20 & 4,8 & 277 & 4,2 \\
\hline
\end{tabular}

Fuente: Encuesta a empresas familiares, 2011

*(Grado de acuerdo en una escala 1 a 5). 
Según estos resultados, la preocupación por el "destino del negocio" y el “orgullo” por la empresa son dos de los aspectos más valorados por el empresariado familiar. En estas variables, se observa un énfasis ligeramente superior entre las mujeres. Como también es algo superior su percepción sobre la implicación de la familia en las "decisiones que afectan al futuro empresarial”, otro de los factores sobre los que el empresariado manifiesta un mayor grado de acuerdo.

En segundo lugar por orden de importancia, el empresariado valora el papel de la familia en las cuestiones que afectan al futuro de la empresa. En este sentido, no existe prácticamente diferencia en dicha percepción en razón de género, aunque las directivas mujeres incidan algo más en la cuestión.

En tercer lugar -con la misma media-, los directivos se pronuncian sobre la influencia de la familia en la empresa (4,6 de media global y en ambos sexos). Se trata de un aspecto importante teniendo en cuenta que la congruencia entre los valores familiares y empresariales constituye uno de los factores de estabilidad en la empresa familiar. Dicha congruencia (expresada en la escala a partir de la afirmación "la familia propietaria comparte los valores de forma similar en el negocio"), concita un importante grado de acuerdo entre los encuestados -en consonancia con la percepción de la familia y la empresa como elementos interconectados- y, también en este caso, esta afirmación es ligeramente superior en el caso de las directivas mujeres (respecto a la de los hombres y a la media global).

El sentido "conciliador" de la familia en la empresa podría explicar la opinión acerca de la delimitación del conflicto en la familia y la empresa. Responden a esta cuestión las percepciones sobre "saber diferenciar los aspectos empresariales de los familiares" (en este caso la diferencia de medias es de 0,4), un supuesto en el que las mujeres encuestadas se pronuncian más claramente que los hombres y, en segundo lugar, la afirmación: "la familia propietaria se ve afectada por los conflictos que se producen en la empresa”, un supuesto sobre el que las mujeres encuestadas de forma consecuente con la diferenciación que establecen entre ambos ámbitos-, manifiestan un bajo grado de acuerdo.

Este mismo sentido conciliador atribuido a la familia en la empresa, explica la percepción sobre su influencia en el clima de la empresa. Esta cuestión -expresada a partir de la afirmación "los valores familiares contribuyen a crear un buen clima en la empresa”-, pone de manifiesto una percepción ligeramente más positiva sobre la influencia familiar en la empresa en el caso de las mujeres empresarias. Se trata de la cuestión en la que la diferencia de medias entre hombres y mujeres es mayor $(0,6)$. De ello se podría inferir una posición de la mujer más cercana a atribuir a la familia la responsabilidad del buen clima en la empresa.

En suma, la percepción de la mujer despunta ligeramente respecto a la de los directivos varones en el valor otorgado a la empresa expresado a partir del orgullo y la defensa de las decisiones empresariales. También su percepción es algo más clara que la del directivo varón en cuanto al papel de la familia como propiciadora de un buen clima en la empresa. Ello se identifica con el papel de la mujer en la salvaguardia de la unidad en su papel de madre/esposa en el ámbito familiar, reafirman- 
do el rol conciliador. Ello no obsta para que perciba una separación entre ambos escenarios entre los que, sin embargo, los valores compartidos son importantes. Los elementos que apuntan a una figura que propende a la conciliación están en consonancia con las investigaciones sobre la mujer en la empresa familiar (Ceja, 2008, Sharma y Nordqvist, 2008; Lansberg, 1995; Rowe y Hong, 2000).

Estos aspectos guardan relación con la socialización en el compromiso, la colaboración y la ayuda en el seno de la familia, elementos susceptibles de condicionar la percepción de la mujer en la empresa familiar y que acaban determinando su visión sobre el negocio y los aspectos estratégicos de ésta. La mujer ha sido educada para ejercer como elemento de cohesión y esta educación se manifiesta en su concepción sobre aspectos tales como su visión sobre el papel de los fundadores en la empresa familiar.

La trasposición de valores familiares firmemente arraigados en la mujer, como el cuidado de las generaciones anteriores y futuras a la empresa, es uno de los resultados que se desprende de este estudio. La posición preferente que concede la mujer al fundador/a se pone de manifiesto en los datos de la tabla 3. Las mujeres directivas estarían "totalmente de acuerdo" en conceder un papel formal preferente al fundador o generación anterior en la empresa familiar una vez realizada la sucesión. Se trata de una cuestión respecto a la que se observa una de las diferencias más significativas de opinión entre hombres y mujeres (4,40 de media de acuerdo con esta afirmación en la escala de 1 a 5 para la mujer y 3,82 en la de los hombres).

La confianza de la mujer en la familia como un importante activo para la empresa es otro de los resultados de este análisis. Tal como se aprecia en la tabla 3, las mujeres se manifiesta de acuerdo con la afirmación: "El negocio es más fuerte cuando la familia está implicada", siendo la media en el grado de acuerdo con este supuesto de 4,35 entre las mujeres y de 3,89 entre los hombres, lo que evidencia una diferencia de género sobre el papel atribuido a la familia en el éxito del negocio.

La voluntad de continuidad y "cuidado" respecto a las generaciones anteriores es uno de los elementos destacados e identificativos del papel concedido al capital social familiar, como también sobre las generaciones futuras. Los resultados sobre esta cuestión permiten identificar el alto grado de acuerdo que concita entre las mujeres (aunque también entre los hombres) la afirmación sobre "dar a conocer cuanto antes el negocio a los descendientes”. En esta opinión se evidencia la voluntad de continuidad y preservación del carácter familiar de la empresa, en consonancia con el papel de conservación y cuidado de las estructuras familiares. Esta voluntad guarda relación con la asimilación por parte de la mujer de un papel conservadora/cuidadora- adquirido en el proceso de socialización.

En otro orden de cosas, también puede destacarse el compromiso como elemento de cohesión en la arquitectura de la empresa familiar. El significado que las mujeres (media 4,35) otorgan a la implicación de la familia y su repercusión en el éxito del negocio es sensiblemente superior al de los hombres (media 3,89). Con ello no puede concluirse en una hipotética comparativa falta de valoración hacia este término por parte del colectivo masculino pero sí, quizás, una relativamente mejor disposición de la población femenina al desarrollo de las relaciones informales que, 
más allá de los aspectos puramente empresariales, tienen como telón de fondo el ámbito psicoemocional.

Tabla 3. Grado de acuerdo sobre el papel generacional en la empresa familiar y sexo*

\begin{tabular}{|l|c|c|c|c|c|c|}
\hline \multirow{2}{*}{} & \multicolumn{2}{|c|}{ Hombre } & \multicolumn{2}{|c|}{ Mujer } & \multicolumn{2}{c|}{ Total } \\
\cline { 2 - 7 } & $(\mathrm{N})$ & Media & $(\mathrm{N})$ & Media & $\mathrm{N}$ & Media \\
\hline $\begin{array}{l}\text { Los descendientes deberían conocer el } \\
\text { negocio cuanto antes }\end{array}$ & 255 & 4,00 & 20 & 4,05 & 275 & 4,00 \\
\hline $\begin{array}{l}\text { Los futuros directivos deberían ser } \\
\text { escogidos de la familia }\end{array}$ & 251 & 2,84 & 20 & 3,10 & 271 & 2,86 \\
\hline $\begin{array}{l}\text { El fundador o anterior generación } \\
\text { deberían tener siempre un papel formal } \\
\text { en la empresa }\end{array}$ & 250 & 3,82 & 20 & 4,40 & 270 & 3,86 \\
\hline $\begin{array}{l}\text { El negocio es más fuerte cuando la } \\
\text { familia está implicada }\end{array}$ & 255 & 3,89 & 20 & 4,35 & 275 & 3,92 \\
\hline
\end{tabular}

Fuente: Encuesta a empresas familiares, 2011.

*(Grado de acuerdo en una escala 1 a 5).

Estos aspectos, sin embargo, no impiden que a la hora de seleccionar futuros directivos no se considere la posibilidad de hacerlo externamente. Según los datos obtenidos, las mujeres estarían sensiblemente más predispuestas que los directivos varones a cubrir puestos a partir de la "cantera" familiar. No obstante, de todas las opciones, ésta es la que concita un menor grado de acuerdo entre ambos colectivos.

En suma, la importancia concedida a la implicación generacional y familiar en el éxito de la empresa apunta hacia la implicación de la mujer en la empresa familiar con valores próximos a aquellos en que se produce su socialización, un aspecto que puede determinar su papel en la gestación del capital familiar en términos de valores, actitudes y motivaciones.

\section{Conclusiones}

La participación de la mujer en puestos directivos en la empresa familiar es todavía minoritaria. No obstante, investigaciones recientes ponen de manifiesto que es superior al de empresas que no tiene este carácter (Mateos et al., 2006; Rodríguez y Rodríguez, 2011), una de las razones se encuentra en que la pertenencia a la familia elimina algunas barreras en el acceso a este tipo de puestos. Ello, sin embargo, no implica una supresión de los obstáculos con los que se encuentra para obtener poder y reconocimiento en el ámbito de la empresa familiar en la que, con frecuencia, su participación -en términos de propiedad-, no se ve equiparada a su implicación en puestos directivos.

El análisis de la empresa familiar a partir de las elementos que componen el familiness permite una aproximación descriptiva al papel de la mujer en las dimensiones de poder, experiencia y cultura que identifican el "capital familiar" de la 
empresa, entendido como los valores, normas y creencias moralmente aceptadas en las que se socializan los miembros de la unidad familiar. De ahí que la introducción de la perspectiva de género resulte especialmente indicada, dado el papel que tradicionalmente adopta la mujer en la socialización familiar.

Respecto a la dimensión poder, si bien la variable género no ha resultado determinante en la dotación de órganos de gobierno como es el Consejo de Administración (presente en la mayoría de empresas, ya estén lideradas por hombres o por mujeres), sí se ha observado una diferencia reseñable en cuanto al grado de implicación de la familia en este órgano directivo, mayor cuando las empresas son dirigidas por mujeres.

En cuanto a la dimensión experiencia, existe una menor proporción de empresas en primera generación dirigidas por mujeres -respecto a aquellas dirigidas por hombres-. En consonancia con la literatura sobre el tema (Dumas, 1998) la mujer adopta un papel secundario en el acceso a la dirección y entra, en todo caso, en una segunda generación o sucesivas.

La dimensión cultural revela que las mujeres empresarias conceden un papel importante a la familia como generadora de valores que contribuyen a la armonía y al buen clima en la empresa. Se trata de una de las variables de identificación de familiness en las que las mujeres inciden con mayor contundencia que los hombres. Esta importancia concedida a la familia se ve reforzada por la inclusión de miembros -familiares- en los órganos de dirección de las empresas dirigidas por mujeres en mayor medida que en aquellas dirigidas por hombres.

Por otra parte, las mujeres evidencian un alto grado de implicación con el futuro de la empresa y los elementos que contribuyen al consenso. Estos elementos las llevan a "entender" y "compartir decisiones" (abundando en esta línea conciliadora), a la vez que suponen un importante acicate para el "orgullo" del negocio familiar.

Ello no es óbice para que éstas se muestren precisas a la hora de discernir entre los espacios de la familia y la empresa y, como consecuencia de ello, no atribuyan a los conflictos empresariales un papel significativo en el deterioro de las relaciones de la familia.

En otras palabras, la mujer es una pieza importante en el modo en que se articula la organización, donde, más allá de los aspectos jerárquicos y funcionales, cobran especial relevancia las relaciones informales en la arquitectura empresarial y afectiva de la empresa. La utilidad que la mujer confiere a los valores familiares en la empresa la confirman como generadora de capital familiar.

En este sentido, el papel tradicional de la mujer como transmisora de valores en la familia y, más concretamente, en el ámbito de la empresa familiar resulta un factor moderador de la implicación de la familia en la empresa o, lo que es lo mismo, la potencialidad de la familia para influir en la empresa (familiness). 
Respecto al papel socializador de la familia sobre la mujer, los resultados apuntan a que la mujer adopta en la empresa familiar estrategias respecto a los recursos humanos conforme a los roles transmitidos en el núcleo familiar. Ello puede explicar que la participación de la mujer en la empresa familiar y de forma más explícita en sus órganos de gobierno y puestos directivos-, adopte una posición secundaria (en ocasiones de hija o esposa) reproduciendo la tesis que la convierte en catalizadora de emociones y propiciadora de consensos (confirmando algunas tesis, como la de Martínez et al. (2007), que aluden a la tipificación de la mujer "ancla") y no tanto una posición de liderazgo. Esta conclusión queda reforzada con los datos sobre participación en la propiedad y en la dirección de la empresa familiar que muestran cómo la posición de la mujer alcanza una mayor equidad respecto al hombre en la propiedad que en la dirección de la empresa familiar. Los resultados están en sintonía con otros estudios sobre este tema (Dumas, 1998).

Las claves de la socialización familiar se proyectan sobre la mujer. Las opiniones de las mujeres empresarias sobre el papel del fundador en la empresa -una vez realizado el proceso de sucesión-, y la implicación de las generaciones futuras, aproximan a la mujer al rol de "cuidadora" de los miembros del clan familiar.

De la misma forma opera una socialización que considera a la mujer, no tanto por su competencia, sino por su contribución a la unidad y la armonía en la familia y en la empresa-, un papel que le lleva a ocupar posiciones secundarias en la dirección empresarial. A su vez, la mujer proyecta y reproduce las claves de socialización en el valor que confiere a los aspectos informales, la familia, el orgullo por el negocio, la preocupación por su destino y los valores familiares como contribución al buen clima empresarial.

De todo ello, se podría concluir que la mujer intensifica el papel de la familia en la empresa. Por tanto, contribuye positivamente desde la perspectiva familiness; no obstante, se ve condicionada por un proceso de socialización que refuerza la división sexual del trabajo y es susceptible de relegarla a un papel de propiciadora de consensos y cuidadora y, por consiguiente, de alejarla del perfil de liderazgo en la empresa familiar.

Con las matizaciones que cabe en este tipo de aproximaciones, estos aspectos parecen indicar cómo la empresa - más exactamente la empresa familiar - se convierte en la representación de una sociedad androcéntrica, donde razón y emoción parecen estar dividas por razón de género convirtiendo a la organización en un "laboratorio" donde esas diferenciaciones se proyectan hacia la sociedad.

Este trabajo, que desde un punto de vista teórico aporta una aproximación exploratoria al estudio del género en el familiness, pretende servir de base para estudios sucesivos que desarrollen esta perspectiva, identificando las diferencias de género y sus resultados sobre la empresa, una limitación que en este análisis ha venido dada por la exigua representación de mujeres 
directivas, que no es sino un referente muestral del particular universo sobre el que se construye la empresa familiar. 


\section{Bibliografía}

Astrachan, Joseph, Klien, S. y Kosmas Smyrnios. 2002. "The F-PEC scale of family influence: A proposal for solving the family business definition problem", Family Business Review, 15 (1): 45-58.

Balbo, Laura. 1978. "La doppia presenza”, Inchiesta, 32: 3-11.

Burke, Ronald J. 2007. "Career Development of Managerial Women: Attracting and Managing Talent», en Diana Bilimoriad y Sandy K. Piderit, eds., Handbook on Women in Business and Management. Edward Elgar Publishing Northampton: Massachusetts.

Carrasco, Antonio y Ramón Sabater. 2009. “Gobierno y organización de la empresa familiar”, en Juan Monreal et al.,ed., La gestión de las empresas familiares: un análisis integral. Madrid: Thomson Reuters.

Carrasquer, Pilar, Teresa Torns, Elisabet Tejero y Alfonso Romero.1998. "El trabajo reproductivo", Papers, 55:95-114.

Carrasquer, Pilar. 2009. "La doble presencia. El trabajo y el empleo femenino en las sociedades contemporáneas.” Tesis Doctoral, Departamento de Sociología, Universidad Autónoma de Barcelona.

Ceja, Lucía. 2008. "El papel de la mujer en la empresa familiar: el reconocimiento de un rol crítico pero a veces invisible”, Newsletter n ${ }^{0}$ 28, Cátedra de Empresa familiar: IESE.

Chrisman, James, Lloyd Steier y Jess H. Chua. 2005. "Sources and consequences of distinctive familiness: An introduction”, Entrepreneurship Theory and Practice, 29 (3): 237 - 247.

Chrisman, James, Lloyd Steier y Jess H. Chua. 2008. "Toward a theoretical basis for understanding the dynamics of strategic performance in family firms", Entrepreneurship Theory and Practice, 32 (6): 935 - 947.

Cibrián, Tania. 2010. "La influencia de la familia: el capital social como fuente para la construcción de recursos y capacidades en la empresa familiar”, VIII Jornada de pre-comunicaciones a congresos de Economía y Administración de Empresas, Barcelona: Universidad Autónoma de Barcelona.

Cole, Patricia M. 1997. “Women in Family Business”, Family Business Review, 10 (4): 353-371.

Coleman, James. S. 1988. "Social Capital in the Creation of Human Capital", American Journal of Sociology, 94: 95-120.

Corona, Juan. 2005. Manual de la empresa familiar. Madrid: DeustoPricewaterhouse Coopers.

Danes, Sharon M. Kathryn Stafford y Johnben.T Loy. 2007. "Family business performance: The effects of gender and management", Journal of business Research, 60 (19): 1058-1069.

Dumas, Colette. 1989. "Understanding of father-daughter and father-son dyads in family-owned businesses”, Family Business Review, 2 (1): 31-46.

Dumas, Colette 1998. "Women's pathways to participation and leadership in the family-owned firm”, Family Business Review, 11 (3): 219 - 228. 
Dumas, Colette 1992. "Integrating the daughter into family business management" Entrepreneurship: Theory and Practice, 16: 41-55.

EPA .2013. http://www.ine.es/prensa/epa_prensa.htm [Consulta 10 de octubre de 2012].

Fitzgerald, Margaret A. y Glenn. Muske. 2002. "Copreneurs: An Exploration and Comparison to Other Family Businesses”, Family Business Review, 15 (1): 1-16.

Gallo, Miguel A. 1995. Empresa familiar: Textos y casos. Barcelona: Editorial Praxis.

Gisbert, María C., María J. Alarcón, M.J. y José M. Gómez. 2009. "La mujer y su papel en la industria. Aproximación al caso del sector del juguete”, Economía Industrial, 372:51-64.

Habbershon, Timothy. G. 2006. "A framework for managing the familiness and agency advantages in family firms", Entrepreneurship: Theory and Practice, 30 (6): 879 - 886.

Habbershon, Timothy. G., Mary Williams, M. y Ian MacMillan. 2003. "A unified perspective of family firm performance", Journal of Business Venturing, 18: 451-65.

Hoffman, James, Mark Hoelscher y Ritch Sorenson. 2006. “Achieving sustained competitive advantage: a family capital theory”, Family Business Review, 19 (2): 135-145.

Innarelli, Cindy M. 1992. "The socialization of leaders in family business: an exploratory study of gender”, Doctoral Dissertation, University of Pittsburgh.

Kellermanns, Franz, Kimberly Eddleston,Tim Barnett, y Allison Pearson. 2008. "An exploratory study of family member characteristics and involvement: Effects on entrepreneurial behavior in the family firm”, Family Business Review, 21 (1): 1-14.

Kets de Vries, Manfred.1996. Family business: Human dilemmas in the family firm. London: International Thomson Business Press.

Heinonen, Jarna y Pekka Stenholm. 2011. "The contribution of women in family business”, International Journal of Entrepreneurship and Innovation Management, 13 (1): 62-79.

Hienerth, Cristoph. y Alexander Kessler. 2006. "Measuring Success in Family Businesses: The Concept of Configurational Fit”, Family Business Review, 19(2): 115-134.

Lansberg, Ivan.1983. "Managing Human Resources in Family Firms: The Problem of Institutional Overlap”, Organizational Dynamics, 12 (1): 39-46.

Lansberg, Ivan 1995. "The Power of the Chief Emotional Officer”, Family Business Magazine, spring, http://www.familybusinessmagazine.com/index.php?/issue/spring_1995/ [Consulta: 12 de noviembre de 2012].

Le Breton-Miller, Isabelle y Danny Miller. 2009. "Why do some family businesses out-compete? Governance, long-term orientations, and sustainable capability", Entrepreneurship: Theory \& Practice, 30: 731-746.

Leach, Peter.1999. La empresa familiar.Barcelona: Granica. 
Lyman Amy, Matilde Salganicoff y Bárbara Hollander. 1985.“Women in Family Business: An Untapped Resource”, en C. E. Aronoff, John L. Ward, ed., Family business sourcebook. Detroit: Omnigraphics.

Martínez, Rocío, María J. Hernández y Juan de la Poza. 2007. “Trayectoria profesional de las hijas en la empresa familiar: de la infancia a la sucesión”, en Juan C. Ayala, coord., Conocimiento, innovación y emprendedores: camino al futuro. Logroño: Servicio de Publicaciones de la Universidad de la Rioja.

Maruani, Margaret. 2002. Trabajo y el empleo de las mujeres. Madrid: Fundamentos.

Mateos, Ruth, Lorenzo Escot, y Ricardo Gimeno.2006. Análisis de la presencia de la mujer en los consejos de administración de las mil mayores empresas españolas. Documento de Trabajo 263: Fundación de Cajas de Ahorro.

Mateos, Ruth, Javier Iturrioz y Ricardo Gimeno.2009. "La participación financiera y el papel de la mujer en la toma de decisiones de las sociedades cooperativas: los consejos de administración”, Revista europea de dirección y economía de la empresa, 18(3): 65 - 82 .

Meroño, Ángel y López, Carolina. 2012. "La empresa familiar y el acceso femenino a la gerencia empresarial” en Juan Monreal y Gregorio Sánchez, coord., El éxito de la empresa familiar. La relación entre negocio y familia. Madrid: Civitas.

Miller, Danny, Isabelle Le Breton-Miller y Barry Scholnick. 2008. "Stewardship vs. stagnation: An empirical comparison of small family and non-family businesses", Journal of Management Studies, 45(1): 51 - 78.

Mustakallio, Mikko, Erkko Autio y Shaker A. Zahra. 2002. "Relational and contractual governande in family firms: effect on strategic decisión making”, Family Business Review, 15: 205-222.

Olson, Patricia D. et. al. 2003. "Impact of family and business on family business sustainability”, Journal of Business Venturing,18(5): 639 - 666.

Ortiz, Pilar, Antonio Carrasco y Ángel Olaz (2009): "Cultura y excelencia en la empresa familiar”, en Juan Monreal et al., ed., La gestión de las empresas familiares: un análisis integral, Madrid: Thomson Reuters.

Papí, Natalia y María J. Frau. 2005. "La conciliación del empleo y del hogar: respuesta y reflejo de una organización del trabajo construida desde la institución del género”, Revista Española de Investigaciones Sociológicas,110: 149-171.

Pearson, Allison, Jon Carr y Jhon Shaw. 2008. "Toward a theory of familiness: A social capital perspective”, Entrepreneurship and Practice, 32 (6): 949-969.

Peris-Ortiz, Marta, Fernando Peris y Domingo Ribeiro.2010. “Capacidades y mujer emprendedora”, Información Comercial Española, 852, 153-165.

Putnam, Robert.1995. "Bowling Alone: America's Declining Social Capital”, Journal of Democracy, 6 (1) 65-78.

Rigby, Mike y Enric Sanchís 2006. "El concepto de cualificación y su construcción social”, Revista Europea de Formación Profesional, 37: 24-35.

Rodríguez, Maribel y Rodríguez, Magdalena. 2011. "La mujer en la empresa familiar”, Revista de Empresas Familiar,1: 17-26 
Rowe, Bárbara y Gong S. Hong. 2000. "The role of wives of family businesses: The paid and unpaid work of woman”, Family Business Review, 13 (1): 1-13.

Sabater, Ramón y Antonio Carrasco. 2009. "Gobierno y organización de la empresa familiar”, en Juan Monreal et al., eds. La gestión de las empresas familiares: un análisis integral, Madrid: Thomson Reuters.

Salganicoff, Matilde. 1990. "Women in family business: Challenges and Opportunities”, Family Business Review, 3 (2): 125-138.

Sharma, Pramodita. 2004. "An Overview of the Field of Family Business Studies: Current Status and Directions for the Future”, Family Business Review, 17 (1): 1-36.

Sharma, Pramodita y Mattias Nordqvist. 2008. "A classification scheme for family firms: From family values to effective governance to firm performance", en Josep Tapies y John L. Ward, eds., Family values and value creation: How do family-owned businesses foster enduring values. New York: Palgrave Macmillan.

Sorenson, Ritch y Leonard Bierman. 2009. "Family capital, family business, and free enterprise”, Family Business Review, 22: 193-195.

Tobío, Constanza. 2002. "Conciliación o contratación: cómo hacen las madres trabajadoras”, Revista Española de Investigaciones Sociológicas, 97: 155-186.

Tobío, Constanza. 2005. Madres que trabajan. Dilemas y estrategias. Madrid: Cátedra.

Tobío, C., Agulló, C., Gómez, S. y Martín, M. 2010. El cuidado de las personas. Un reto para el siglo XXI. Barcelona: Fundación "La Caixa”.

Torns, Teresa. 2005. "De la imposible conciliación a los permanentes malos arreglos”, Cuadernos de Relaciones Laborales, 23 (1): 15-33.

Vadnjal, Jaka y Blaz Zupan. 2009. "The role of women in family businesses", Economic and Business Review, 11 (2): 159-177.

Vera, Carolina F. y Michelle Dean. 2005. "An examination of the challenges daughters face in family business succession”, Family Business Review, 18 (4): 321-346. 


\section{ANEXO - CUESTIONARIO}

\section{GENERAL}

1. Indique el número medio de empleados que ha tenido su empresa en el último año:...............................empleados

2. En comparación con sus competidores más directos, su empresa...:

1. Es más rentable

2. Tiene un mejor comportamiento en ventas

3. Presenta mayor independencia financiera

4. Cuenta con mayor reputación y mejor imagen

\begin{tabular}{|lllll|}
\hline \multicolumn{2}{|l}{$\begin{array}{l}\text { Total } \\
\text { Desacuerdo }\end{array}$} & \multicolumn{2}{|r|}{$\begin{array}{r}\text { Total } \\
\text { Acuerdo }\end{array}$} \\
\hline 1 & 2 & 3 & 4 & 5 \\
1 & 2 & 3 & 4 & 5 \\
1 & 2 & 3 & 4 & 5 \\
1 & 2 & 3 & 4 & 5 \\
\hline
\end{tabular}

3. Indique, con respecto al director general / gerente de la empresa...:

\begin{tabular}{|llc|}
\hline 1. Sexo: & $\square$ Hombre & $\square$ Mujer \\
\hline 2. Nivel de estudios: & $\square$ Primarios / Secundarios & $\square$ Universitarios \\
\hline 3. Antigüedad en el cargo: & $\ldots \ldots \ldots \ldots \ldots \ldots$ años & \\
\hline
\end{tabular}

4. En relación con la internacionalización de su empresa...:

1. ¿Qué porcentaje representan las exportaciones sobre el total de ventas del último ejercicio:

\begin{tabular}{lll}
\hline 2. ¿A qué zonas exporta su empresa?: & $\square$ UE \\
Resto & $\square$ Iberoamérica
\end{tabular}

5. Indique la importancia de los siguientes límites para la internacionalización Nada Muy de su empresa:

1. El mercado nacional ofrece más oportunidades de crecimiento

2. Su producto/servicio está orientados al consumidor nacional.

3. El nivel de tecnología resulta insuficiente (para los mercados internacionales).

4. Los recursos financieros resultan insuficientes (para acceder a los mercados internacionales)

5. Hay resistencia de la propiedad y/o dirección a la internacionalización

Importante Importante

6. ¿Tiene la empresa carácter familiar? (una o varias familias forman parte de la propiedad y/o dirección):

\section{ORIENTACIÓN CULTURAL Y FAMILIAR}

7. Piense en el principal accionista de su empresa, así como en los familiares del mismo, y responda:

\begin{tabular}{|c|c|c|c|}
\hline 1. Proporción de propiedad en manos de: & \multicolumn{3}{|c|}{$\begin{array}{l}\text { (a) Familia del principal accionista } \\
\text { (b) Otros propietarios no familiares } \%\end{array}$} \\
\hline 2. ¿'Tiene su negocio consejo de administración? & \multicolumn{2}{|c|}{$\begin{array}{l}\square \text { No } \\
\square \text { Si } \quad \text { (a) ¿Qué \% de sus miembros son familiares? }\end{array}$} & $\%$ \\
\hline 3. ¿Qué \% de puestos directivos está ocupado por mien & ros de la familia? & $\%$ & \\
\hline 4. ¿Qué generación controla la propiedad del negocio? & & $\square$ Fundadora $\square$ Otra: ¿Cuál? & \\
\hline 5. ¿Qué generación dirige actualmente la compañía? & & $\square$ Fundadora $\square$ Otra: ¿Cuál? & \\
\hline 6. ¿El director general / gerente es miembro de la fam & ropietaria? & $\square$ No & \\
\hline
\end{tabular}

8. ¿Existe en su empresa...?:

\begin{tabular}{|lll}
\hline 1. Protocolo familiar: & $\square$ No & $\square \mathrm{S}$ \\
\hline 2. Consejo de familia: & $\square$ No & $\square \mathrm{S}$
\end{tabular}

$\square$ No

$\mathrm{Si}$

9. Indique su grado de acuerdo con las siguientes afirmaciones. "La familia propietaria...:

1. Tiene influencia en el negocio

2. Comparte valores similares en el negocio

3. Se siente orgullosa del negocio

4. Se preocupa por el destino del negocio

5. Entiende y defiende las decisiones que afectan al futuro de la empresa

6. Sabe diferenciar los aspectos empresariales de los familiares

7. Se ve afectada por los conflictos que se producen en la empresa

8. Con sus valores familiares contribuye a generar un buen clima en la empresa

10. Indique su grado de acuerdo con las siguientes afirmaciones:

1. Los descendientes deberían conocer el negocio cuanto antes

2. Los futuros directivos deberían ser escogidos de la familia

3. El fundador o anterior generación deberían tener siempre un papel formal en la empresa

4. El negocio es más fuerte cuando la familia está implicada

\section{Política y Spciedad}

Penale la importancia de los siguientes aspectos para su empresa familiar:

2014 . 1 indes sociafes desarromlas por relaciones personales de los miembros familiares

2. La existencia de códigos de conducta compartidos por los miembros familiares

\begin{tabular}{|c|c|c|c|c|}
\hline \multicolumn{3}{|c|}{$\begin{array}{l}\text { Total } \\
\text { Desacuerdo }\end{array}$} & \multicolumn{2}{|c|}{$\begin{array}{r}\text { Total } \\
\text { Acuerdo }\end{array}$} \\
\hline 1 & 2 & 3 & 4 & 5 \\
\hline 1 & 2 & 3 & 4 & 5 \\
\hline 1 & 2 & 3 & 4 & 5 \\
\hline 1 & 2 & 3 & 4 & 5 \\
\hline 1 & 2 & 3 & 4 & 5 \\
\hline 1 & 2 & 3 & 4 & 5 \\
\hline 1 & 2 & 3 & 4 & 5 \\
\hline 1 & 2 & 3 & 4 & 5 \\
\hline \multicolumn{3}{|c|}{$\begin{array}{l}\text { Total } \\
\text { Desacuerdo }\end{array}$} & \multicolumn{2}{|c|}{$\begin{array}{r}\text { Total } \\
\text { Acuerdo }\end{array}$} \\
\hline 1 & 2 & 3 & 4 & 5 \\
\hline 1 & 2 & 3 & 4 & 5 \\
\hline 1 & 2 & 3 & 4 & 5 \\
\hline 1 & 2 & 3 & 4 & 5 \\
\hline \multicolumn{3}{|c|}{$\begin{array}{l}\text { Nada } \\
\text { Importante }\end{array}$} & \multicolumn{2}{|c|}{ Importante } \\
\hline 1 & 2 & 3 & 4 & 5 \\
\hline 1 & 2 & 3 & 4 & 5 \\
\hline
\end{tabular}




\section{FISCALIDAD EMPRESA FAMILIAR}

12. En relación con la fiscalidad de su empresa...:

\begin{tabular}{|ll|}
\hline 1. ¿Conoce los beneficios fiscales de las empresas familiares?: & $\square$ No (Pase a P14) \\
\hline 2. ¿Cree que son suficientes?: & $\square$ No \\
\hline
\end{tabular}

13. ¿Qué beneficios fiscales le parece que se deberían incorporar al sistema fiscal español?

\begin{tabular}{|l|l|}
\hline 1. Deducciones adicionales a la formación profesional del empresario y sus familiares más cercanos que trabajen & \\
en la empresa en el Impuesto sobre Sociedades & $\square$ \\
2. Deducciones adicionales a la inversión en I+D+i en el Impuesto sobre Sociedades & $\square$ \\
3. Implantar el régimen especial de empresas de reducida dimensión a todas las empresas familiares & $\square$ \\
4. Deducción en el IRPF a los familiares por las aportaciones para la capitalización de la empresa familiar & $\square$ \\
5. Reducción del tipo de gravamen en el Impuesto sobre Sociedades & $\square$ \\
6. Otros (indique & $\square$ \\
cuál):................................................................................................................. & $\square$ \\
\hline
\end{tabular}

\section{RECURSOS HUMANOS E INNOVACIÓN}

\begin{tabular}{|c|c|c|c|c|c|}
\hline \multirow{2}{*}{$\begin{array}{l}\text { 14. La política de de recursos humanos de su empresa se caracteriza por...: } \\
\text { 1. La rigurosidad en el proceso de selección }\end{array}$} & \multicolumn{3}{|c|}{\begin{tabular}{|l|l} 
Total \\
Desacuerdo
\end{tabular}} & \multicolumn{2}{|c|}{$\begin{array}{r}\text { Total } \\
\text { Acuerdo } \\
\end{array}$} \\
\hline & 1 & 2 & 3 & 4 & 5 \\
\hline 2. La selección de empleados en función de habilidades requeridas para el puesto & 1 & 2 & 3 & 4 & 5 \\
\hline 3. La selección de empleados según potencial de desarrollo en la empresa & 1 & 2 & 3 & 4 & 5 \\
\hline 4. La existencia de una política formativa integral & 1 & 2 & 3 & 4 & 5 \\
\hline 5. La disponibilidad de formación continua para empleados & 1 & 2 & 3 & 4 & 5 \\
\hline 6. El fácil acceso de los nuevos contratados a acciones formativas & 1 & 2 & 3 & 4 & 5 \\
\hline 7. La estrecha relación entre el rendimiento del empleado y la retribución que recibe & 1 & 2 & 3 & 4 & 5 \\
\hline 8. El pago de incentivos individuales (bonus o primas) & 1 & 2 & 3 & 4 & 5 \\
\hline 9. El pago de incentivos de grupo (reparto de beneficios) & 1 & 2 & 3 & 4 & 5 \\
\hline
\end{tabular}

15. En su empresa...:

1. Se adopta una visión conservadora cuando se toman decisiones importantes

2. Existe una tendencia a apoyar proyectos cuyos resultados esperados son ciertos

3. Sus operaciones generalmente son previamente testadas

4. Sus operaciones generalmente se caracterizan como de bajo riesgo

5. Se han introducido gran cantidad de productos/servicios nuevos al mercado

6. Se han introducido muchas modificaciones a los productos/servicios existentes

7. Constantemente se buscan nuevos productos/servicios

8. Se introducen nuevos productos/servicios que causan gran impacto en la industria.

\begin{tabular}{|ccccc|}
\hline $\begin{array}{l}\text { Total } \\
\text { Desacuerdo }\end{array}$ & & \multicolumn{2}{r|}{$\begin{array}{r}\text { Total } \\
\text { Acuerdo }\end{array}$} \\
\hline 1 & 2 & 3 & 4 & 5 \\
1 & 2 & 3 & 4 & 5 \\
1 & 2 & 3 & 4 & 5 \\
1 & 2 & 3 & 4 & 5 \\
1 & 2 & 3 & 4 & 5 \\
1 & 2 & 3 & 4 & 5 \\
1 & 2 & 3 & 4 & 5 \\
1 & 2 & 3 & 4 & 5 \\
\hline
\end{tabular}

\section{CAPITAL FINANCIERO}

16. ¿Ha solicitado su empresa financiación a las entidades de crédito en los últimos 3 años?:

\begin{tabular}{|llll}
\hline 1. $\square$ No & \\
\hline 2. $\square \mathrm{No}$, porque a pesar de necesitarlo no lo conseguiría & \\
\hline 3. $\square \mathrm{Si}$ & (a) $\dot{\boldsymbol{i}} \mathrm{Ha}$ obtenido la financiación solicitada?: $\square$ No & $\square$ Si, pero menos de lo solicitado \\
\hline
\end{tabular}

17. Con relación a las condiciones de financiación bancaria durante los 3 últimos años:

1. Las garantías y avales que necesita para acceder a la financiación han aumentado

2. El coste de la financiación se ha incrementado

3. El plazo exigido de la devolución se ha acortado

18. Con respecto a las entidades financieras (bancos, cajas de ahorro y cooperativas de crédito) con las que trabaja su empresa?:

1. ¿Con cuantas entidades financieras trabaja?:

2. Del total de su deuda, ¿qué porcentaje mantiene con su principal entidad financiera?

3. ¿Cuántos años lleva trabajando con su principal entidad financiera?:

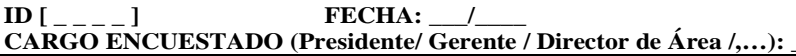

